\title{
POTENTIAL OF AN AUTOMATIC GROUNDING ZONE CHARACTERIZATION USING WRAPPED INSAR PHASE
}

\author{
Alessandro Parizzi \\ Remote Sensing Technology Institute German Aerospace Center (DLR) \\ Münchenerstraße 2082234 Weßling; alessandro.parizzi@dlr.de
}

\begin{abstract}
The work deals with the identification and the characterization of the grounding zone area using InSAR data. The idea is to point towards a methodology that minimizes the role of the operator and provides results with performance that can be mathematically described using input parameters. The approach uses the information of the interferometric phase gradient to follow the path of the grounding zone and fit them using a physical model that describes the ice bending. The approach is tested on more than $300 \mathrm{~km}$ grounding zone comparing also the results with existing products.
\end{abstract}

Index Terms - InSAR; Deformation Measurements; Cryosphere; Grounding Line

\section{INTRODUCTION}

The characterization of the transition from floating to grounded ice is a very important information for glaciologists. Its behavior and evolution supports the understanding of the dynamic processes of the ice sheets. A systematic monitoring is therefore necessary to support scientists working in this field.

Since many years researchers deploy instrumentation to derive measurements in order to locate and understand the temporal evolution of the ice-sea boundary[1] [2]. The rise of the remote sensing satellites gave the perspective of a more systematic and global mapping of areas that would be anyway difficult and expensive to survey. The strong sensitivity of InSAR to vertical displacements allows a clear identification of the transition highlighting the ice deformation due to the tidal cycle. In [3] the potential of interferometry for the study of grounding areas has been shown identifying their position and measuring their movements. Over many years the coverage and the systematic of the interferometric measurements has been increased reaching a global maps of the Grounding Lines (GL)[4].

However the interferometric data are typically used to identify the grounding area manually marking the position of the Grounding Line directly on the computed interferograms. This work studies an approach that is able to identify and measure the Grounding Zone (GZ) in a more automatic fashion. Since the wrapped interferometric phase is inherently

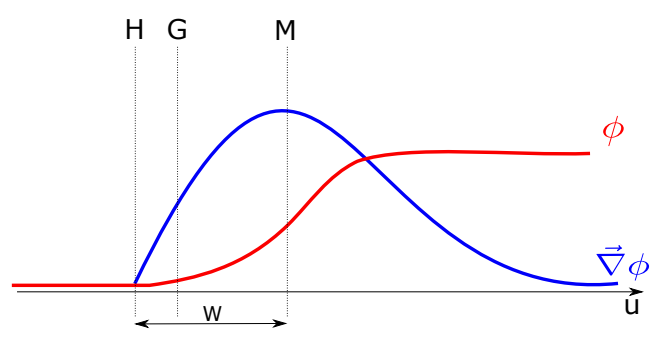

Fig. 1: Schematic of the Grounding Area displaying phase and phase gradient profile. $\mathrm{H}$ is the position of the hinge Line, $\mathrm{G}$ of the Grounding Line and $\mathrm{M}$ is the maximum of the gradient.

a measure of the deformation gradient InSAR data can be used analogously to the tiltmeter measurement also avoiding the phase unwrapping that could harm the robustness of the approach. The study relies on the physical model proposed in [5] and applied in [6] but using the phase gradients instead of the absolute phase. The gradient information allows both to follow the geometry of the GZ and to estimate the model parameters.

\section{METHODOLOGY}

The model proposed in [5] describes the displacement due to the tide cycle of the floating ice w.r.t the grounded ice. The idea of the proposed algorithm is to be able to spatially follow the grounding zone on the wrapped SAR interferogram and characterize it fitting the physical model in [5]. As previously mentioned in Section 1 the approach is based on the spatial gradients of the interferometric phase $\vec{\nabla} \phi$ that are computed estimating the main fringe frequency window-wise on the complex SAR interferogram. Being $(r, a)$ the range and azimuth coordinates in radar geometry, the interferogram is a complex number $z(r, a)$. Considering the interferometric phase varying by several wavelengths within the scale of the deformation pattern, it is possible to perform a local linear approximation of the deformation phase. Hence in a given point $\left(r_{0}, a_{0}\right)$ the phase $\phi$ of the interferogram $z=A e^{j \phi}$ can be substituted by its first order Taylor approximation: 


$$
\phi \approx \frac{4 \pi}{\lambda}\left(\frac{\partial \delta_{s}}{\partial r}\left(r-r_{0}\right)+\frac{\partial \delta_{s}}{\partial a}\left(a-a_{0}\right)\right)+\phi_{0}
$$

where $A$ is the product of master and slave reflectivity and $\delta_{s}=\boldsymbol{\delta}^{T} \boldsymbol{s}$ is the projection of the displacement vector $\boldsymbol{\delta}$ along the line of sight $s(\mathrm{LoS})$. Equation 1 shows how, under the linear approximation hypothesis, the deformation gradients are basically a scaling of the interferogram fringe frequencies [7]. Therefore the gradient measurements $\vec{\nabla} \delta_{s}$ can be hence estimated from the single look complex data performing a frequency estimation [8], [9]. Multi-looking and sub-sampling are anyhow implicit in the fringe frequency estimation since the computation is done window-wise. This allows theoretically identifying gradients that would not any more visible at the multi-looked interferogram level due the resolution reduction .

The deformation gradient provides basically two information the amplitude $\Gamma=\left\|\vec{\nabla} \delta_{s}(u)\right\|$ and the angle $\eta=\left\langle\vec{\nabla} \delta_{s}\right.$. $\eta$, represents the direction of maximum slope and $\frac{\pi}{2}+\eta$ identifies the local direction of the GZ path. The gradient data can be hence re-sampled along the direction $u$ defined as the direction rotated by $\eta$ w.r.t. the reference axes. Now exploiting the model in [5] it is possible to model the data as follows:

$$
\Gamma=\frac{\partial \delta_{v}(u)}{\partial u}=\frac{2 \beta \Delta}{1+e^{-\pi}} \sin (\beta(u-H)) e^{-\beta(u-H)} \quad \forall u \geq H
$$

where $\Delta$ is tide the displacement, $\beta$ is a shape factor describing the width of the bending area, $H$ is the hinge line position along the axis $u$. The inversion of the GZ parameters can be hence performed bypassing the phase unwrapping ${ }^{1}$ by fitting in Equation 2 the estimated gradient $\Gamma$.
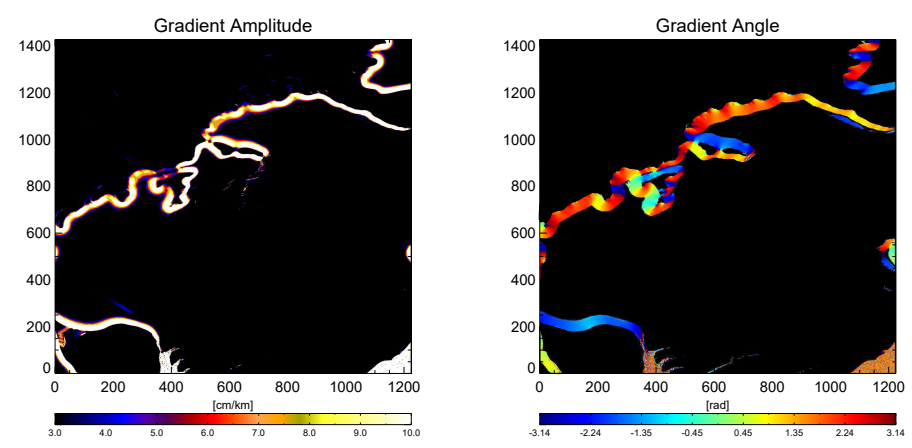

Fig. 2: Estimated Phase gradients displayed in terms of Amplitude and Angle.

The inversion derives first the position of the so called Hinge Line (HL) and $\beta$ that provide a characterization of the

\footnotetext{
${ }^{1}$ For the sake of precision it would be rather to say that the procedure implicitly solve the phase ambiguities supported by the model
}

GZ. $\beta$ is a shape factor that describes the curve determining the width of the GZ. This is visible from the analytic expression of the distance $W_{\text {peak }}$ between the HL and the maximum of the deformation gradient that identify the center of the GZ see Figure 1.

$$
W_{\text {peak }}=\frac{\pi}{4 \beta}
$$

Iterating the described steps on the gradient map the fringe belt can be followed estimating the parameters of the GZ as in Figure 3.
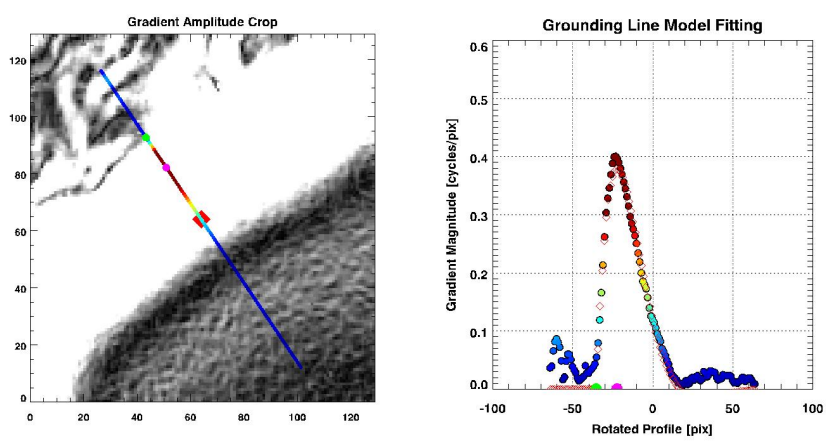

Fig. 3: Model fitting in the gradients map

\section{RESULTS}

Real data experiments have been carried out in order to test the potential automation of the procedure. Two test sites have been selected testing both qualitatively and quantitatively the accuracy of the estimated positions. Two ERS Tandem pairs (1 day temporal baseline) and one Sentinel one Double Differences [4] over the Antarctica have been processed and the described algorithm have been applied on the computed interferograms. A semi-automatic implementation has been tested in order to verify the described approach. An a-priori has been defined over the interferogram. Starting from that the algorithm finds the gradient direction for each point intersecting the a-priori, interpolates the gradient along such line and fits the model to the gradient amplitude retrieving the estimated position for the HL (black dots). The vector of the "raw" retrieved positions is finally geocoded.

\subsection{Schirmacher Area using ERS-Tandem}

In the first test site considered it is possible to notice a quite complex geometry of the grounding line that follows the coastline in all gulfs and peninsulas. Figure 4 shows a the result of the estimation displaying the retrieved positions of the HL and the distance between HL and maximum gradient point color-coded. This information can compactly show 
a model-based characterization of the GZ since it displays position and dimension of the GZ.

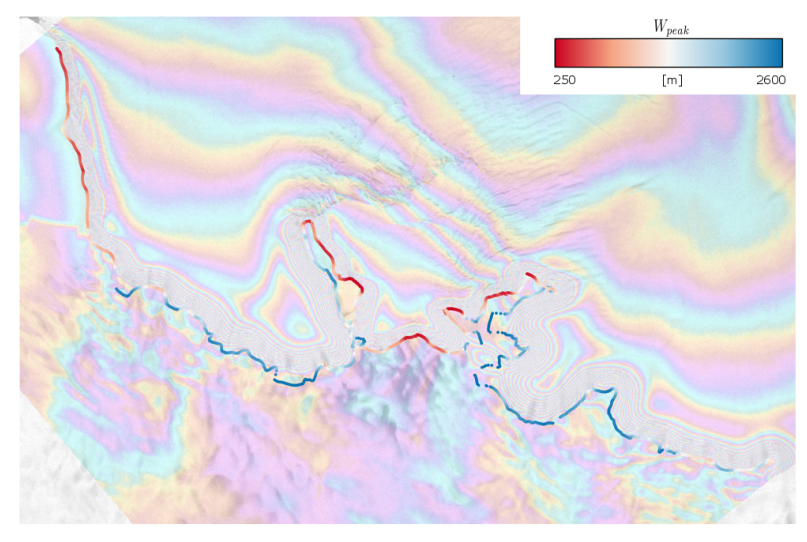

Fig. 4: The overview of the whole processed area showing both the HL positions and the $W_{\text {peak }}$ distance color-coded.

\subsection{Inter-comparison with MEaSURES data set}

However a quantitative comparison of the derived product with existing products is necessary in order to verify the trustworthiness of the estimated positions. Therefore another area of interest in the Princess Raghild Coast has been selected. The same ERS Tandem data used to generate the MEaSUREs product have been processed. The HL position has been then calculated using the same strategy described for the first test site. As already visible from the overview Figure 5 the positions are very similar nonetheless the result has been compared with MEaSUREs computing the local distance of each extracted point from the reference product. The local distance between the two product has been calculated computing for each point of the distance from the slope that fits the three closest points of the other product. Proceeding in this way it was possible to collect a vector of local distances to be used to derive some statistics. The histogram and the statistics of the differences are plotted in Figure 5. The deviation of the position is about $115 \mathrm{~m}$ but drops to $82 \mathrm{~m}$ if robustly computed using MAD. Such numbers are compatible with accuracies measured in [4]. A not negligible bias $(>150 \mathrm{~m})$ is however detectable between the different position. Looking more in detail to Figure 5 is visible that the proposed method positions are located systematically more in the inland. This should probably be related to a different definition of the position between the MEaSUREs (GL) product and the model used in this study (HL), see Figure 1.

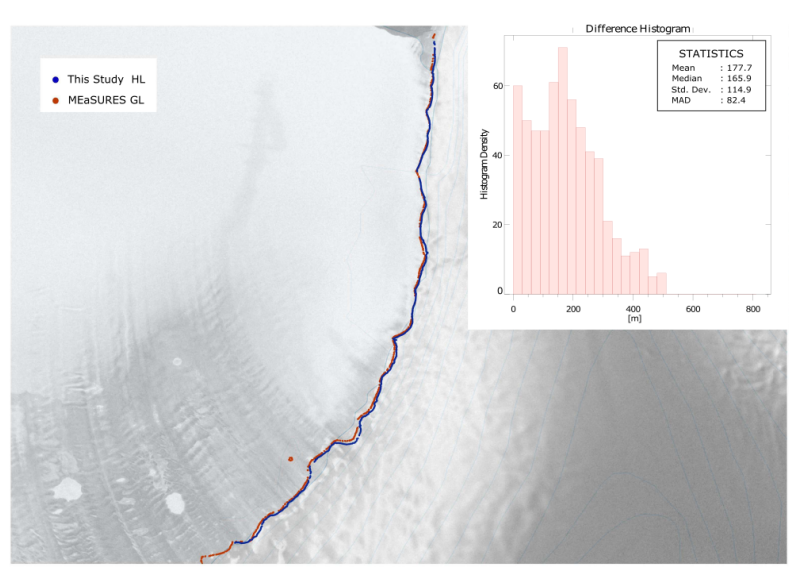

Fig. 5: Result of the comparison on the second test site, extracted products (MEaSUREs in red, this study in blue) and the histogram and the statistics of the local distances.

\subsection{Schirmacher Area using Sentinel-1 Double Differ- ences}

Nowadays the Sentinel-1 mission is able provide interferometric data on regular basis with a global coverage. Therefore this is the most suitable sensor to address the problem of Grounding Zones monitoring using InSAR. However the 6 days revisit time does not allow to neglect the ice motion specially in proximity of Glaciers. Therefore, in this case, the use of interferometric double differences have to be considered [4]. The approach can be extended to gradients having two interferograms w.r.t a master image temporally located in the middle of the three acquisitions. Given that it would be possible to delete the constant horizontal motion component simply adding the gradients vector retrieved from the tow interferograms.

$$
\vec{\nabla} \phi_{d d}=\vec{\nabla} \phi_{-1}+\vec{\nabla} \phi_{1}
$$

where $\phi_{-1}$ and $\phi_{1}$ represent the two interferograms. The results of the extracted map are shown in Figure 6, the derived Hinge Line position is shown in Figure 7

\section{CONCLUSIONS}

The proposed method shows potential in characterizing the GZ using directly wrapped SAR interferograms pointing towards a more automatic approach. The implementation developed in this work starts from a coarse a-priori roughly derived by the gradient map.Starting from that the relations between the physics of the problem and the measurement approach (InSAR) are used to derive a fine mapping of the $\mathrm{HL}$ 

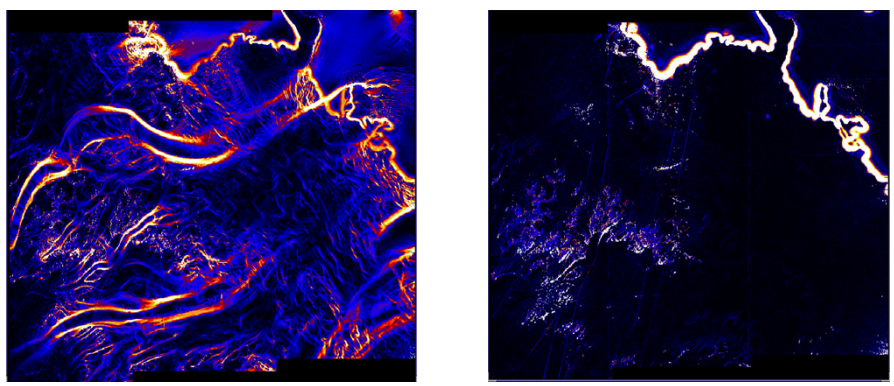

Fig. 6: Interfeograms (left) and double differences (right) phase gradients amplitude

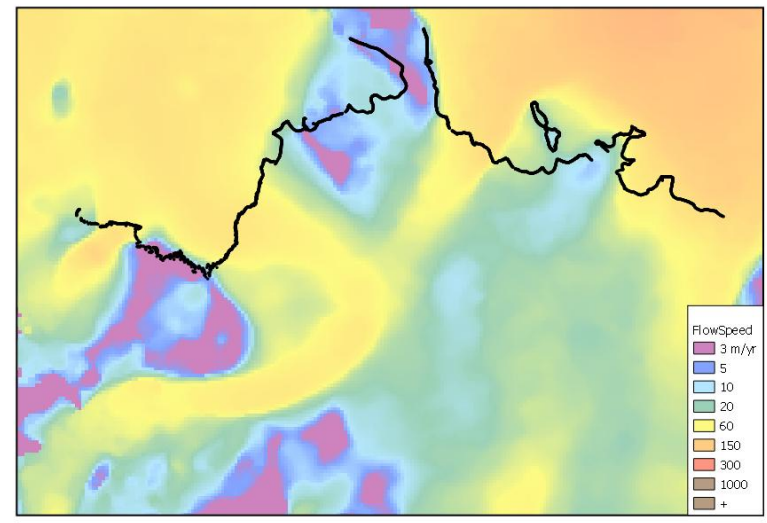

Fig. 7: Hinge Line derived from Sentinel-1 double differences, plotted over the Ice Velocity map [10].

and a measurements of the GZ width. This requires consistently less effort than the typical manual mapping using the fringe belt: for a single interferogram only few benchmarks roughly located in the fringe belt are necessary. This work is focused on the investigation of a general a approach using InSAR data. In order to do that the model in [5] [6] has been used. The choice is mainly related to its easy analytic expression, nonetheless other more sophisticated models can be " "plugged", into this framework [11]. Since most of the problems could derive from the nature of real data also different test sites and data have been considered analyzing the retrieved results. Moreover a quantitative comparison with existing GL products has been also carried out. In the proposed test sites more than $300 \mathrm{~km}$ GZ have been processed showing besides a good agreement with the MEaSUREs data set. Finally the method has been applied also to Sentinel-1 data extending it for the use of double differences.

\section{REFERENCES}

[1] S. N. Stephenson, "Glacier Flexure and the Position of Grounding Lines: Measurements By Tiltmeter on Rutford Ice Stream, Antarctica," Annals of Glaciology, vol. 5, pp. 165-169, 1984.

[2] A. M. Smith, "The use of tiltmeters to study the dynamics of antarctic ice-shelf grounding lines," Journal of Glaciology, vol. 37, no. 125, pp. 51-58, 1991.

[3] Richard M. Goldstein, Hermann Engelhardt, Barclay Kamb, and Richard M. Frolich, "Satellite Radar Interferometry for Monitoring Ice Sheet Motion: Application to an Antarctic Ice Stream," Science, vol. 262, no. 5139, pp. 1525-1530, 1993.

[4] E. Rignot, J. Mouginot, and B. Scheuchl, "Antarctic grounding line mapping from differential satellite radar interferometry," Geophysical Research Letters, vol. 38, no. 10, 2011.

[5] G. Holdsworth, "Flexure of a Floating Ice Tongue," Journal of Glaciology, vol. 8, no. 54, pp. 385-397, 1969.

[6] Eric Rignot, "Hinge-line migration of Petermann Gletscher, north Greenland, detected using satelliteradar interferometry," Journal of Glaciology, vol. 44, no. 148 , pp. 469-476, 1998.

[7] A. Parizzi and W. Abdel Jaber, "Estimating Strain and Rotation From Wrapped SAR Interferograms," IEEE Geoscience and Remote Sensing Letters, vol. 15, no. 9, pp. 1367-1371, Sept 2018.

[8] S. Kay and R. Nekovei, "An efficient two-dimensional frequency estimator," Acoustics, Speech and Signal Processing, IEEE Transactions on, vol. 38, no. 10, pp. 1807-1809, Oct 1990.

[9] A. Monti Guarnieri and S. Tebaldini, "ML-Based Fringe-Frequency Estimation for InSAR,' IEEE Geoscience and Remote Sensing Letters, vol. 7, no. 1, pp. 136-140, Jan 2010.

[10] J. Mouginot, E. Rignot, and B. Scheuchl, "ContinentWide, Interferometric SAR Phase, Mapping of Antarctic Ice Velocity," Geophysical Research Letters, vol. 46, no. 16, pp. 9710-9718, 2019.

[11] Christian T. Wild, Oliver J. Marsh, and Wolfgang Rack, "Unraveling InSAR Observed Antarctic Ice-Shelf Flexure Using 2-D Elastic and Viscoelastic Modeling," Frontiers in Earth Science, vol. 6, pp. 28, 2018. 